Thr ee- di mensi onal fl ow vi sual i zat i on syst em by hol ogr aphi c i nt erfer omet ry

\begin{tabular}{|l|l|}
\hline 著者 & UEDA Masahi r o, M YASAKA Kenj i , OKUNO Shi n- i chi \\
\hline $\begin{array}{l}\text { j our nal or } \\
\text { publ i cat i on t i t l e }\end{array}$ & Opt i cal and Quant um El ect r oni cs \\
\hline vol une & 21 \\
\hline number & 5 \\
\hline page r ange & $409-414$ \\
\hline year & $1989-09$ \\
\hline URL & ht t p: //hdl . handl e. net /10098/1468 \\
\hline
\end{tabular}




\title{
Three-dimensional flow visualization system by holographic interferometry
}

\author{
MASAHIRO UEDA, KENJI MIYASAKA, SHIN-ICHI OKUNO \\ Faculty of Education, Fukui University, Fukui 910, Japan
}

A holographic system has been developed to visualize a three-dimensional fluid flow. The system consists principally of a thermoplastic film, a monochrome video camera, a microcomputer with image-processing capability and a printer. The system makes it possible to measure a slow flow. Two examples of such, the bimodal and mixed-mode convection flows within a Bénard cell, are presented.

\section{Introduction}

The continuing development of laser-based diagnostic techniques offers considerable potential for advancing research on fluid dynamics because of their non-intrusive nature. In the past such techniques have generally been limited to a single point measurement. Laser Doppler anemometry provides data representing discrete samples of the flow velocities in time and space.

In order to study fluid motion such as a convective flow, full-field information gathering is essential. New techniques are developed to fulfil the requirements for high spatial and temporal resolution. Laser-based techniques for full-field, instantaneous velocity measurements generally rely on two major optical characteristics: interference and diffraction. Wavefront interference methods, including holographic interferometry and speckle methods, measure the change of the optical path length through a test cell. Although speckle interferometry allows the use of lower-resolution photographic emulsion than does the holographic interferometry and reduces information loss due to incidental vibration, its maximum velocity range is lower [1-3].

We have previously developed a method for a full-field two-dimensional velocity measurement by holographic interferometry [4] and have successfully applied to the fluid motion measurement of a convection in a Bénard cell [5]. Such two-dimensional (2-D) flow velocity distributions of each section are superimposed to construct a 3-D flow distribution. The measurement of the fluid motion in this system has, however, been restricted to a stationary flow due to a long access time, far too long for a sectional measurement, because the system includes a conventional photographic process.

The purpose of the present study has been to develop a practical system for a 3-D flow velocity measurement which is applicable for the measurement of a spatially complicated flow motion. The main improvement of the present system over the original is the use of a thermoplastic film [6], which replaces the holographic plate, and a monochrome video camera and a microcomputer replace the photographic storage of the image data. 


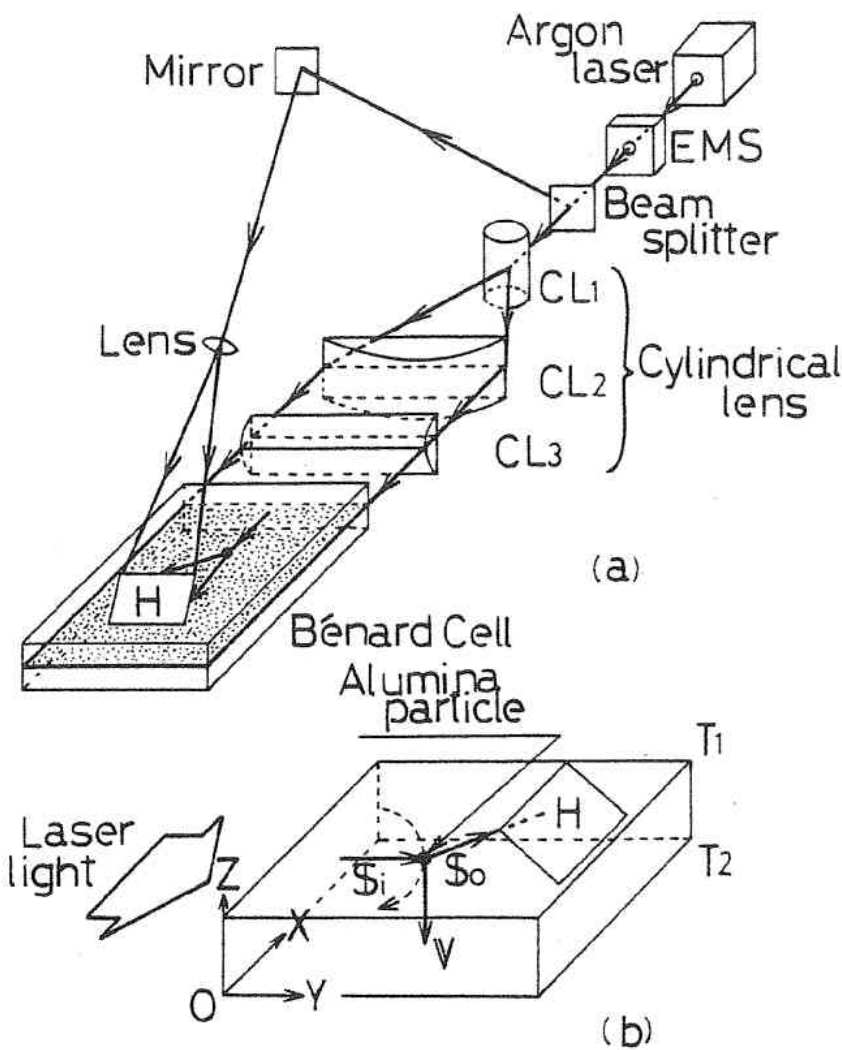

Figure 1 Optical arrangement for measuring flow velocity distribution by holographic interferometry.

\section{Hologram-recording system}

A detailed description of the system has been given in a previous paper [4]. A brief outline of the optical arrangement used is shown in Fig. 1a. Optics were constructed on a vibrationinsulated optical table $(2000 \mathrm{~mm} \times 4000 \mathrm{~mm})$ which was vibration free for frequencies above $50 \mathrm{~Hz}$. A $2 \mathrm{~W}$ continuous-mode argon laser was used as a light source. The multiple pulses with a duration of 3 to $10 \mathrm{~ms}$ and variable separation, $\Delta t$, were then obtained by using an electromagnetic shutter (EMS). The sheet-like light with a thickness of approximately $0.05 \mathrm{~mm}$ was formed through cylindrical lenses $\mathrm{CL}_{1}, \mathrm{CL}_{2}$ and $\mathrm{CL}_{3}$. The position of the light sheet was easily changed in the $Z$-direction by shifting lens $\mathrm{CL}_{3}$ up and down.

A schematic of a rectangular Bénard cell is shown in Fig. $1 \mathrm{~b}$. The upper and lower plates are maintained at a constant temperature, $T_{1}$ and $T_{2}$, respectively, by circulating water from temperature-controlled baths. The cell is filled with glycerol in which fine alumina particles of approximately $1 \mu \mathrm{m}$ are suspended as light scatterers. The convection flow, called Bénard convection, is caused by an appropriate temperature difference, $\Delta T=T_{2}-T_{1}$. The motion of the scatterers is assumed to be identical to the fluid flow, then the fluid flow can be visualized using holographic interferometry.

The measurable component of the flow velocity, $V_{\mathrm{m}}$, obtained from the reconstructed fringe pattern can be expressed as

$$
V_{\mathrm{m}}=\frac{m \lambda}{|\underline{S}| \Delta t}
$$

where $S=S_{\mathrm{i}}-S_{\mathrm{o}}, S_{\mathrm{i}}$ and $S_{\mathrm{o}}$ being the unit vectors in the propagation direction of the sheetlike incident light and in the observation direction, respectively, as shown in Fig. 1b, $\lambda$ is the wavelength of the laser light and $m$ is an integer which corresponds to the fringe number. The velocity component, $V_{\mathrm{m}}$, is a close approximation to the vertical component. 
The error is estimated as a few per cent, since the angle between observation direction and the incident one was about $45^{\circ}$ in this experiment. The maximum of $V_{\mathrm{m}}$ and the maximum vertical velocity component are then obtained at about the mid-height of the cell.

A multiple-exposed hologram was recorded on a thermoplastic film instead of on a holographic plate. This reduces the processing time to approximately $50 \mathrm{~s}$. It was therefore possible to record the object information in the film and observe the reconstructed image $50 \mathrm{~s}$ after the exposure.

\section{Image-processing system}

In a holographic experiment a holographic plate has been conventionally used to store a fringe pattern because fringes locate very closely to each other and require a very high recording resolution. Although the conventional holographic plate such as Agfa-Gevart $10 \mathrm{E} 56$ meets this requirement, its handlings such as cutting and mounting on an optical holder are not as convenient as plastic photographic film. Developing the plate is also time-consuming, hours being needed before the reconstructed image can be viewed.

In this study a new technique was developed to solve the inconvenience of the conventional method by combining the thermoplastic recording technique [6] and the image-acquisition system. The thermoplastic recording techniques uses a plastic film, Rottenkolber PT1000HS, consisting of a thermoplastic base and photoconductor. A uniform charge is applied on the film before exposure, and light energy redistributes the charges through the photoconductor during the exposure, making a difference in the electric fields between lighted and unlighted portions. After the exposure heat is applied to the film to cause deformation which is linearly related to the light intensity; a phase hologram appears. This thermoplastic recording technique reduces the processing time for obtaining a hologram; however, the film material used in this study has a serious drawbacks, in that the information recorded in the film disappears in a few days as the film deformation is restituted gradually. Thus, we need to transfer a recorded fringe pattern or a reconstructed image to a non-volatile image storage before the film loses its fringe patterns. Although a number of non-volatile methods are available, none provides enough resolution to store a fringe pattern, having sufficient only for conventional image recording. Therefore, we store a reconstructed image rather than the fringe pattern by using a video camera and a microcomputer.

The image-processing system used in this study is shown in Fig. 2. A reconstructed image was captured by a monochrome video camera having 400 horizontal scanning lines, and its

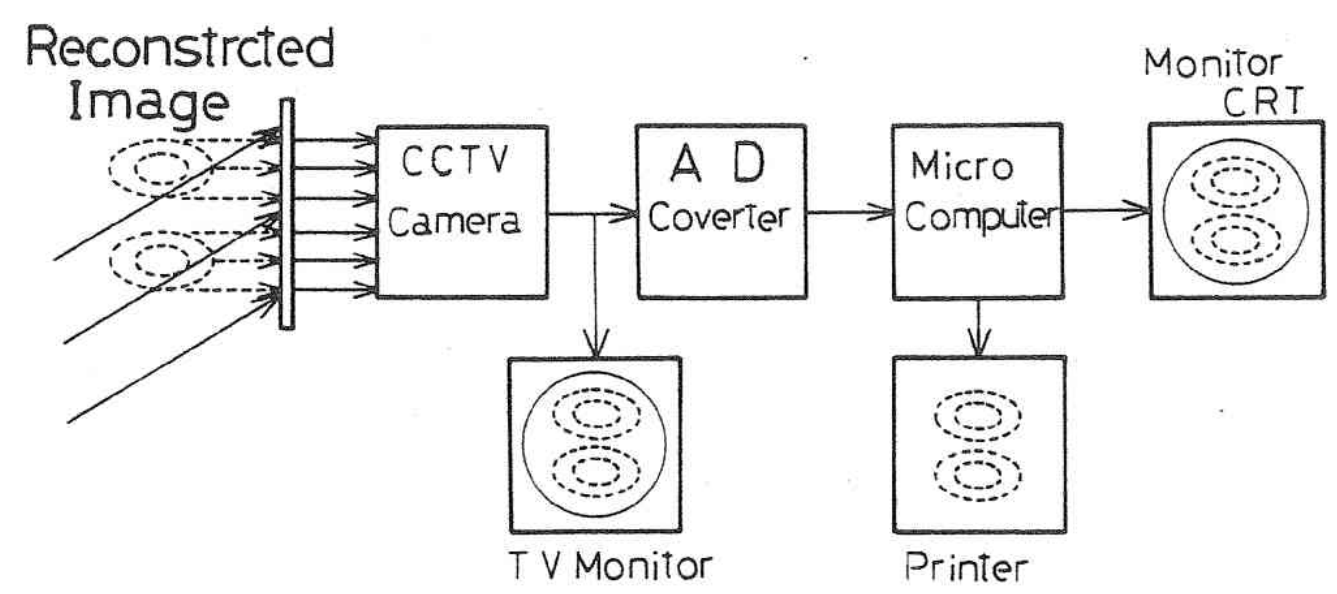

Figure 2 Image-processing system used for the experiments. 
signal was sent to an analogue-to-digital (A/D) converter to digitize. The A/D converter used in this study was a 5-bit flush type designed for the real-time video signal processing, digitizing into 64 gradations in $1 / 60 \mathrm{~s}$. The digitized data of the reconstructed image were then transferred to the computer memory for further processing. The computer has an $80286 \mathrm{CPU}, 3$ Mbyte memory and two $640 \times 400$ 16-colour graphic planes. The image data transferred to the computer was averaged and smoothed in order to enhance the image quality, producing a reconstructed image with an excellent contrast in a spatially uniformed background. A 3-D velocity distribution was constructed from the processed images of which a hard copy was obtained through a bit-image printer.

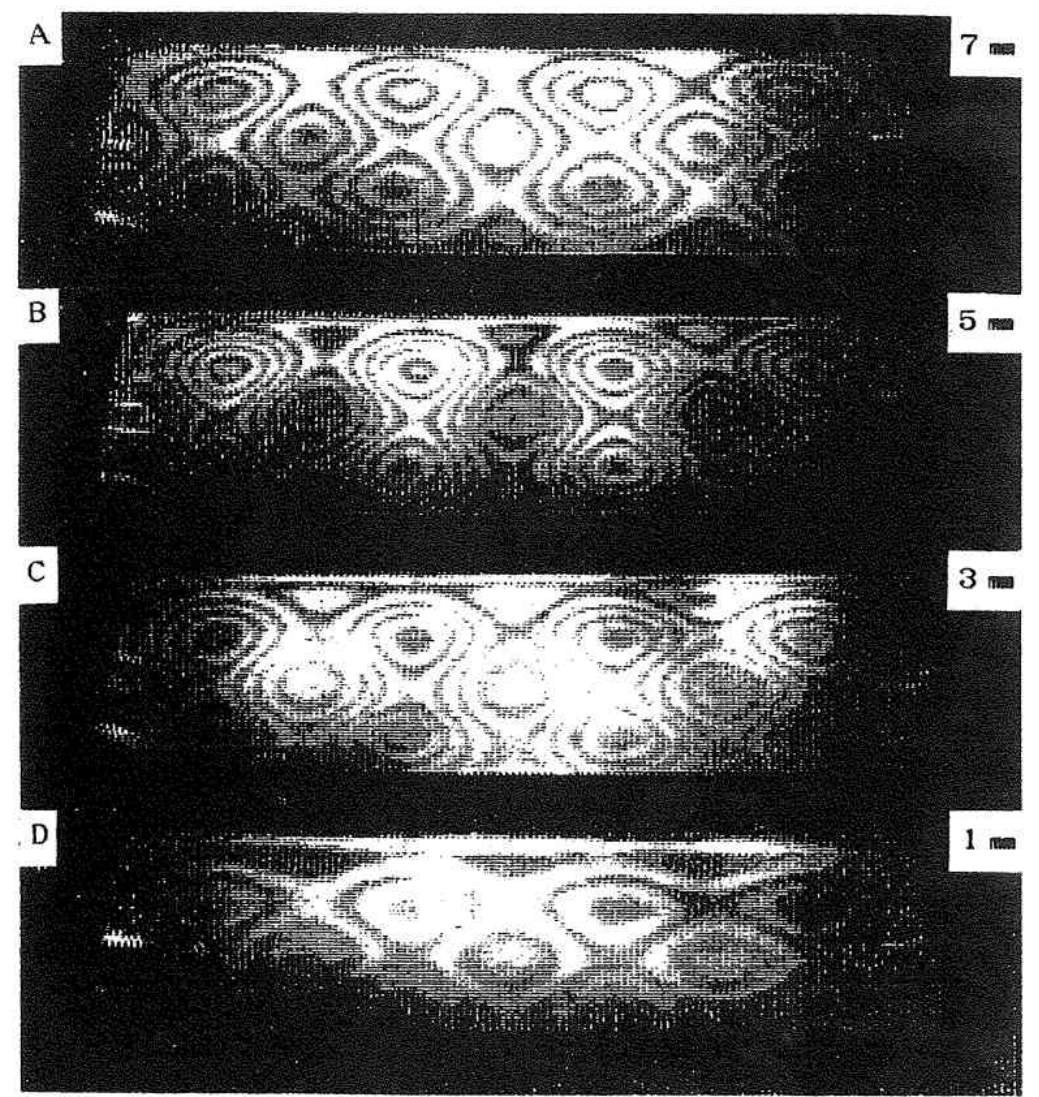

Figure $3 \mathrm{~A}$ set of reconstructed images on the planes of (a) $Z=7 \mathrm{~mm}$, (b) $Z=5 \mathrm{~mm}$, (c) $Z=3 \mathrm{~mm}$ and (d) $Z=1 \mathrm{~mm}$. In all of these images, one fringe corresponds to a velocity change of $87 \mu \mathrm{m} \mathrm{s}^{-1}$.

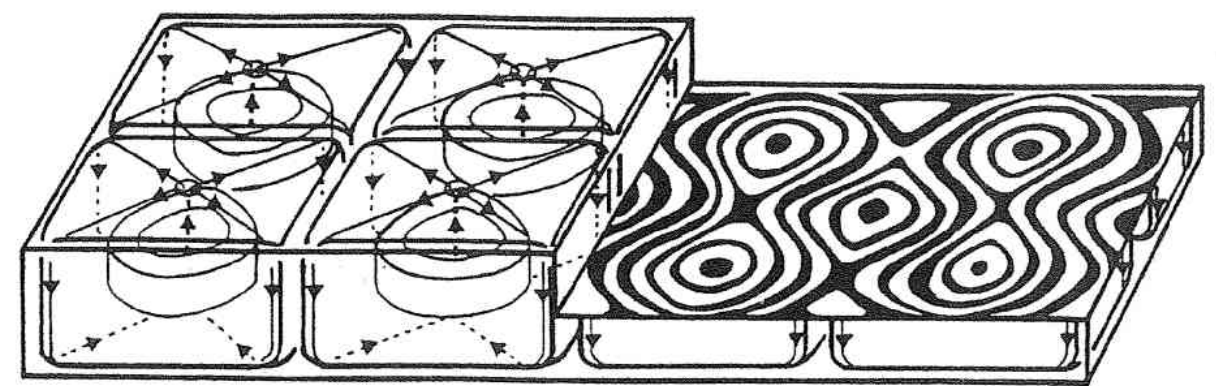

Figure 4 An outline of the 3-D flow distribution obtained from the image in Fig. 3. The flow is expected as a square cell convection in fluid dynamics. 
(a)
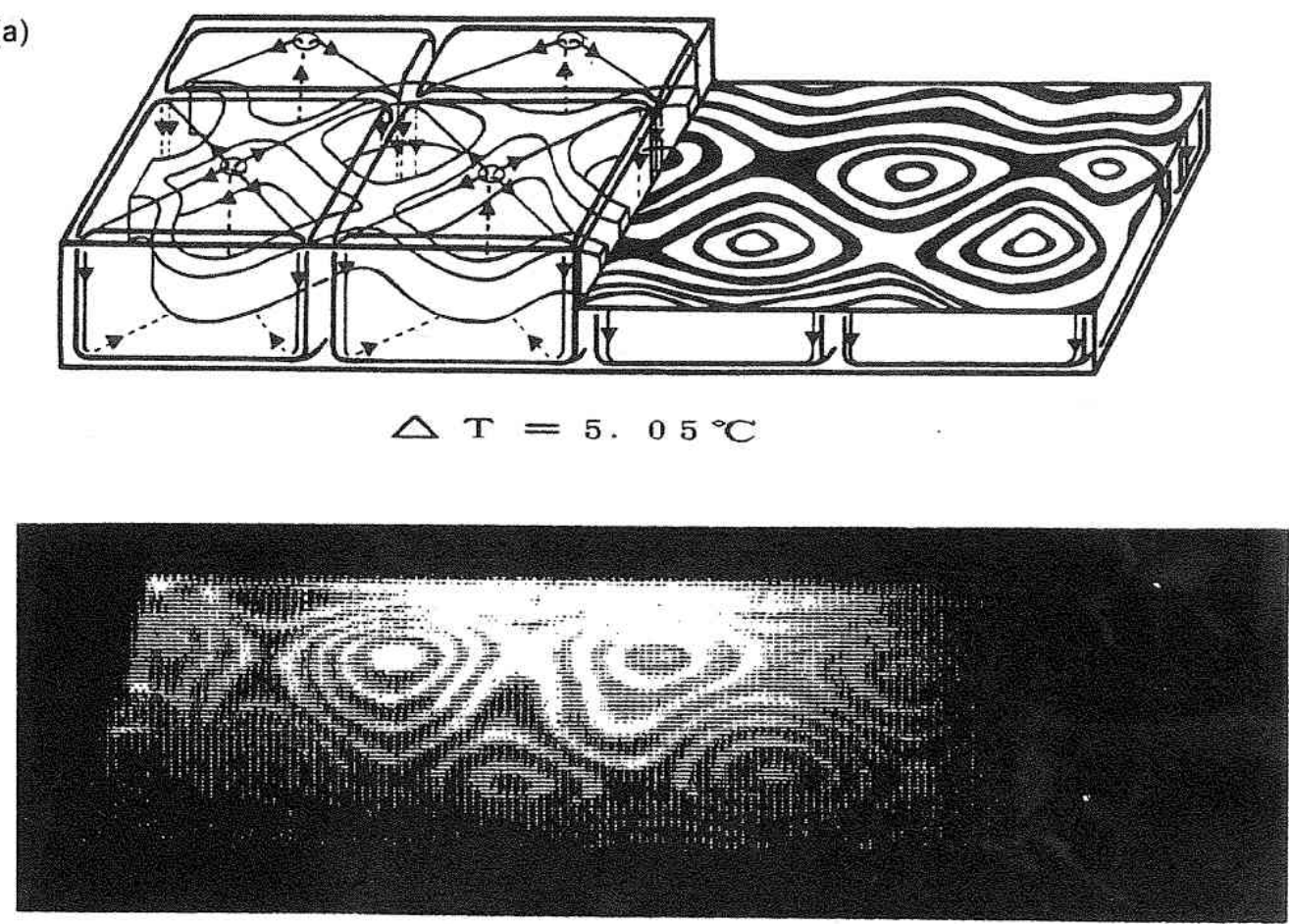

(b)
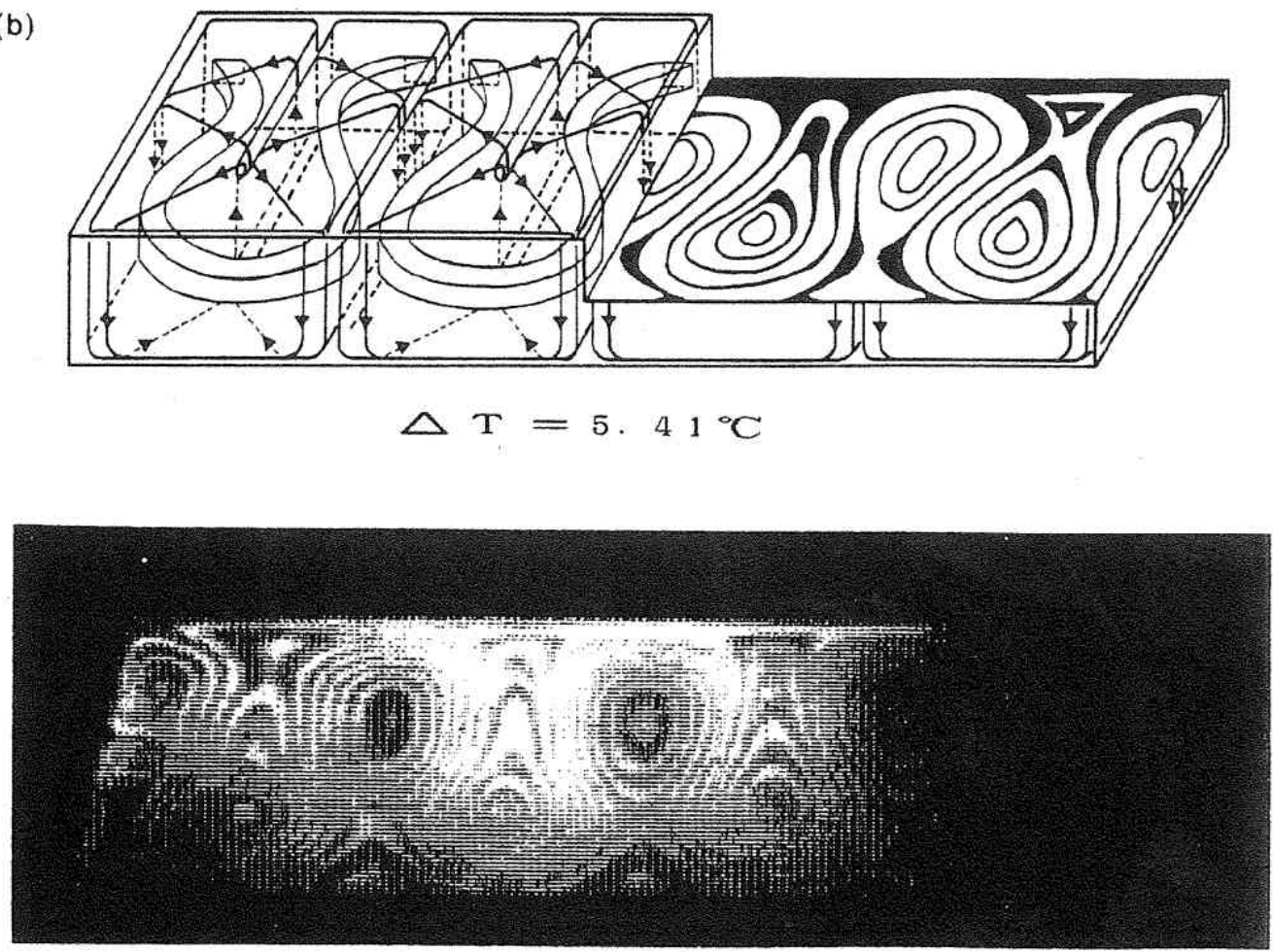

Figure 5 Other examples of the 3-D flow distribution and the typical 2-D image of the flow on the plane of $Z=5 \mathrm{~mm}$. These are expected as (a) bimodal convection and (b) mixed-mode convection (parallel-roll convection plus square cell convection). 


\section{Results}

Fig. 3 shows a set of reconstructed images on planes of $Z=7,5,3$ and $1 \mathrm{~mm}$. The temperature difference, $\Delta T$, was $6.25^{\circ} \mathrm{C}$ and each fringe corresponds to a velocity change of $87 \mu \mathrm{m} \mathrm{s}^{-1}$. Fig. 4 is a constructed 3-D flow pattern from these images. About 50 s were required to record one sectional 2-D image on the thermoplastic film. Four or five sectional 2-D images are usually sufficient for a 3-D full-field construction. Therefore, only a few minutes were needed to record and construct the $3-\mathrm{D}$ velocity distribution. This means that we could measure a velocity field within a few minutes by the use of thermoplastic film and microcomputer. Fig. 5 shows other examples of the typical 2-D images and the constructed 3-D flow pattern for $\Delta T=5.05^{\circ} \mathrm{C}$ (Fig. 5a) and $\Delta T=5.41^{\circ} \mathrm{C}$ (Fig. 5b). Those two flows are expected to be a bimodal convection and a mixed-mode convection, respectively, in fluid dynamics [7]. Many other types of convection were observed, and some knowledge about the phase transition in the fluid motion was also obtained from the experiments. These results will be presented elsewhere.

\section{Conclusions}

A holographic 3-D flow visualization system has been developed by means of a thermoplastic film, a video camera, a microcomputer and a printer replacing a conventional holographic plate. It requires only a few minutes to construct a 3-D velocity field from several 2-D velocity images, and can therefore be successfully applied to a 3-D measurement of a convection flow. The system has been used to visualize a convection flow in a Bénard cell, and some interesting convection flow patterns such as a bimodal convection flow and a mixed-mode convection flow have been found as well as a parallel-roll convection flow.

\section{References}

1. F. P. CHIANG and R. M. JUANG, Appl. Opt. 15 (1976) 2199.

2. P. G. SIMPKINS and T. D. DUDDERAR, J. Fluid Mech. 89 (1978) 665.

3. R. MEYNART, Proc. Soc. Photo-Opt. Instrum. Engng 210 (1979) 25.

4. M. UEDA, K. YAMADA, C. YAMAGUCHI and Y. HARADA, Appl. Opt. 21 (1982) 3269.

5. M. UEDA, M. HOSONO and Y. HARADA, Optik 63 (1983) 363.

6. R. MORAW, Proc. Fourth Int. Cong. Repro. and Inf. (1975) p. 13.

7. E. L. KOSCHMIEDER, Adv. Chem. Phys. 26 (1974) 177. 